\title{
АВТОМАТИЗИРОВАННЫЕ СИСТЕМЫ УПРАВЛЕНИЯ
}

\author{
УДК [621.314.26+621.316.94+621.317.757]:621.311.1
}

\begin{abstract}
Александр Аркадьевич Николаев
ФГБОУВО «Магнитогорский государственный технический университет имени Г.И. Носова», кандидат технических наук, заведующий кафедрой автоматизированного электропривода и мехатроники, Россия, Магнитогорск, e-mail: aa.nikolaev@magtu.ru
\end{abstract}

Ильдар Галиевич Гилемов

ФГБОУВО «Магнитогорский государственный технический университет имени Г.И. Носова», аспирант кафедры автоматизированного электропривода и мехатроники, Россия, Магнитогорск, e-mail: gilemov.ig@outlook.com

\section{Михаил Викторович Буланов}

ФГБОУВО «Магнитогорский государственный технический университет имени Г.И. Носова», аспирант кафедры автоматизированного электропривода и мехатроники, Россия, Магнитогорск, e-mail: bulanovv.m.v@gmail.ru

\section{Оценка влияния режимов работы электроприводов прокатного стана с ПЧ-АВ на качество напряжения питающей сети 10 кВ}

\section{Авторское резюме}

Состояние вопроса. Современные электроприводы прокатных станов строятся на базе преобразователей частоты с активными выпрямителями. Работа таких преобразователей оказывает негативное влияние на систему внутризаводского электроснабжения. В целях улучшения качества напряжения в активных выпрямителях применяют специальные алгоритмы широтно-импульсной модуляции. Как правило, все исследования влияния электроприводов с ПЧ-АВ на питающую сеть осуществляются на упрощенных математических моделях, не учитывающих возможные резонансные явления в системе электроснабжения и режимы работы электроприводов. В связи с этим целью работы является оценка допустимости такого подхода.

Материалы и методы. Тренды основных параметров электроприводов стана за цикл прокатки записаны с помощью программного обеспечения IBA PDA. Полученные зависимости аппроксимированы и использованы в комплексной имитационной модели системы внутризаводского электроснабжения с учетом параметров питающей сети, кабельных линий, понизительных трансформаторов, электроприводов стана и других электроприемников цеховых подстанций. Основным допущением при имитационном моделировании является учет нагрузок остальных электроприемников в виде постоянных величин.

Результаты. Проведено исследование влияния режимов работы электропривода прокатного стана на качество электроэнергии распределительной сети 10 кВ системы внутризаводского электроснабжения металлургического предприятия. Установлено, что коэффицциент суммарных гармонических составляющих $K_{U}$ на секциях 10 кВ главной понизительной подстанции металлургического предприятия изменяется нелинейным образом в динамических режимах разгона и торможения электроприводов, характер изменения коэффрициента $K_{U}$ также зависит от наличия резонансных явлений в частотной характеристике питающей сети среднего напряжения.

(c) Николаев А.А., Гилемов И.Г., Буланов М.В., 2021

Вестник ИГЭУ, 2021, вып. 5, с. 41-50. 
Выводы. Полученные результаты доказывают необходимость учета режимов работы электроприводов при исследовании влияния электроприводов с ПЧ-АВ на качество напряжения питающей сети при проведении экспериментальных исследований на действующем оборудовании предприятий, а также при выполнении теоретических исследований на математических моделях. Адекватность имитационной модели ранее подтверждена экспериментальными данными и предыдущими исследованиями.

Ключевые слова: электромагнитная совместимость, преобразователь частоты, активный выпрямитель, широтно-импульсная модуляция, резонанс токов, режим работы электропривода

\section{Alexander Arkadyevich Nikolaev}

Nosov Magnitogorsk State Technical University, Candidate of Engineering Sciences (PhD), Head of Automatic Electric Drive and Mechatronics Department, Russia, Magnitogorsk, e-mail: aa.nikolaev@magtu.ru

\section{II'dar Galievich Gilemov}

Nosov Magnitogorsk State Technical University, Postgraduate student of Automatic Electric Drive and Mechatronics Department, Russia, Magnitogorsk, e-mail: gilemov.ig@outlook.com

\section{Mikhail Viktorovich Bulanov}

Nosov Magnitogorsk State Technical University, Postgraduate student of Automatic Electric Drive and Mechatronics Department, Russia, Magnitogorsk, e-mail: bulanovv.m.v@gmail.ru

\section{Assessment of influence of rolling mill FC-AR electric drive operation mode on $10 \mathrm{kV}$ supply network voltage quality}

\section{Abstract}

Background. Advanced rolling mill electric drives are based on frequency converters (FC) with active rectifiers (AR). Operation of such converters has negative impact on the in-plant power supply system. To improve the voltage quality of active rectifiers, special algorithms of pulse-width modulation algorithms are used. As a rule, the study of the effect of electric drives with FC-AR on the supply network are carried out based on simplified mathematical models that do not consider possible resonance phenomena in the power supply system and operating mode of electric drives. Thus, the aim of the paper is to assess the acceptability of such an approach. Materials and methods. The trends of the main parameters of the rolling mill electric drives have been recorded during the rolling cycle using the IBA PDA software. The obtained dependencies values have been approximated and applied in a complex simulation model of the in-plant power supply system. It considers the parameters of the supply network, cable lines, step-down transformers, rolling mill electric drives and other electrical receivers of the shop substations. The main assumption in simulation modeling is to consider the loads of other electrical receivers in the form of constant values.

Results. The authors have studied the effect of the operating modes of the rolling mill electric drive on the electric power quality in the $10 \mathrm{kV}$ distribution network of the in-plant power supply system of a metallurgical enterprise. It is found that the total harmonic distortion $K_{U}$ at $10 \mathrm{kV}$ sections of the main step-down substation of a metallurgical enterprise is changing in a nonlinear manner in the dynamic modes of acceleration and deceleration of electric drives. The nature of the coefficient $K_{U}$ also depends on the presence of resonance phenomena in the frequency response of the medium voltage supply network.

Conclusions. The obtained results prove the need to consider the operating modes of electric drives when studying the influence of electric drives with FC-AR on the quality of the supply network voltage. in case we conduct experimental research using the equipment of the enterprises, as well as during theoretical research using mathematical models. The adequacy of the simulation model has previously been confirmed by experimental data and previous studies.

Key words: electromagnetic compatibility, frequency converter, active rectifier, pulse width modulation, current resonance, electric drive operation mode

DOI: 10.17588/2072-2672.2021.5.041-050

Введение. В последние десятилетия широкое применение в электроприводах станов горячей и холодной прокатки получили многоуровневые преобразователи частоты с активными выпрямителями
(ПЧ с АВ) [1-5]. Широкому использованию AB в мощных электроприводах способствовал ряд их преимуществ перед диодными и тиристорными выпрямителями. Первым является возможность работы без потребле- 
ния реактивной мощности и обеспечение рекуперации электрической энергии в питающую сеть в тормозных режимах электропривода [6, 7]. Вторым преимуществом является улучшение гармонического состава потребляемого тока в низкочастотном диапазоне за счет применения специальных алгоритмов ШИМ (ШИМ с удалением выделенных гармоник или ШИМ с подавлением выделенных гармоник) [2, 4, 8-11]. Однако практический опыт эксплуатации показал, что ПЧ с АВ не лишены недостатков. На ряде металлургических предприятий после внедрения ПЧ с АВ наблюдались проблемы $[12,13]$ с функционированием электрооборудования, выходы из строя преобразователей частоты, функционирующих параллельно мощным ПЧ с АВ главных электроприводов прокатного стана, аварийные отключения ПЧ с АВ при провалах и несимметрии напряжения питающей сети, ухудшение качества напряжения во внутризаводской распределительной сети.

Проблема, связанная с электромагнитной совместимостью мощных ПЧ с АВ с питающей сетью на одном металлургическом предприятии, рассмотрена в [5]. На данном предприятии после реконструкции четырехклетьевого стана холодной прокатки были введены в работу 5 мощных электроприводов клетей и натяжной моталки, построенные на базе трехуровневых ПЧ с АВ серии ACS6000 и синхронных двигателей. Исследования показали, что сильные искажения напряжения обусловлены взаимным влиянием емкостей кабельных линий и индуктивностей токоограничивающих реакторов на секциях главной понизительной подстанции (ГПП). Также было выявлено, что используемые алгоритмы ШИМ АВ не оптимальны. Были предложены новые таблицы углов переключения силовых ключей АВ. Таблицы были рассчитаны с учетом исключения из гармонического состава потребляемых преобразователями токов гармоник, попадающих в зону резонансов в частотной характеристике сети относительно секций главной понизительной подстанции. Имитационное моделирование показало значительный положительный технический эфффект от предложенного алгоритма. Однако эти исследования проводились только для двух статических режимов работы ПЧ и не учитывали динамические режимы работы электропривода.
В работах, посвященных исследованию влияния ПЧ с $\mathrm{AB}$ на качество напряжения питающей сети [14-18], как правило, представлены исследования только для статического режима работы электропривода. Динамические режимы работы электропривода не учитываются. Также при имитационном моделировании часто инвертер и синхронный или асинхронный двигатель упрощенно представляются в виде источника тока, эквивалентного номинальной нагрузке $[4,14,18]$.

В связи с вышеизложенным возникла необходимость в проведении детальных исследований качества напряжения электрической сети внутризаводского электроснабжения во всех режимах работы электроприводов прокатного стана.

Методы исследования. Установленные на четырехклетьевом стане холодной прокатки ПЧ фрирмы ABB ACS6000 получают питание с секций распределительной подстанции (РП) (рис. 1). При этом реализованы два различных схемотехнических решения: электропривод клети 1 и моталки выполнен по 6-пульсной схеме с использованием двухобмоточных трансформаторов мощностью 12 и 4,8 МВт соответственно, а клетей 2-4 - по 12-пульсной с использованием трехобмоточных трансформаторов мощностью по 12 МВт. Электроприводы клетей 1 и 3 получают питание с секции 1 РП, клетей 2,4 и моталки - с секции 2. Секция 1 РП запитана от секции 2 главной понизительной подстанции ГПП (фидер 1) через токоограничивающий реактор. Секция 2 РП получает питание от секции 1 ГПП (фридер 21) также через токоограничивающий реактор.

На первом этапе работы [5] были проведены экспериментальные исследования качества напряжения в различных точках распределительной сети $10 \mathrm{kB}$, а именно на секциях РУ-10 кВ ГПП и РП. На рис. 2 представлены измеренные мгновенные значения фразных и линейных напряжений на секциях ГПП и РП и их гармонический состав при работе клетей стана. Суммарный коэффицциент гармонических составляющих напряжения на секциях ГПП $K_{u}$ при параллельной работе секций с питанием от одного понизительного трансформатора 110/10 кВ составляет 7,14 \% (рис. 2,б). Значимыми гармониками являются гармоники с номерами 23, 29, 37. 


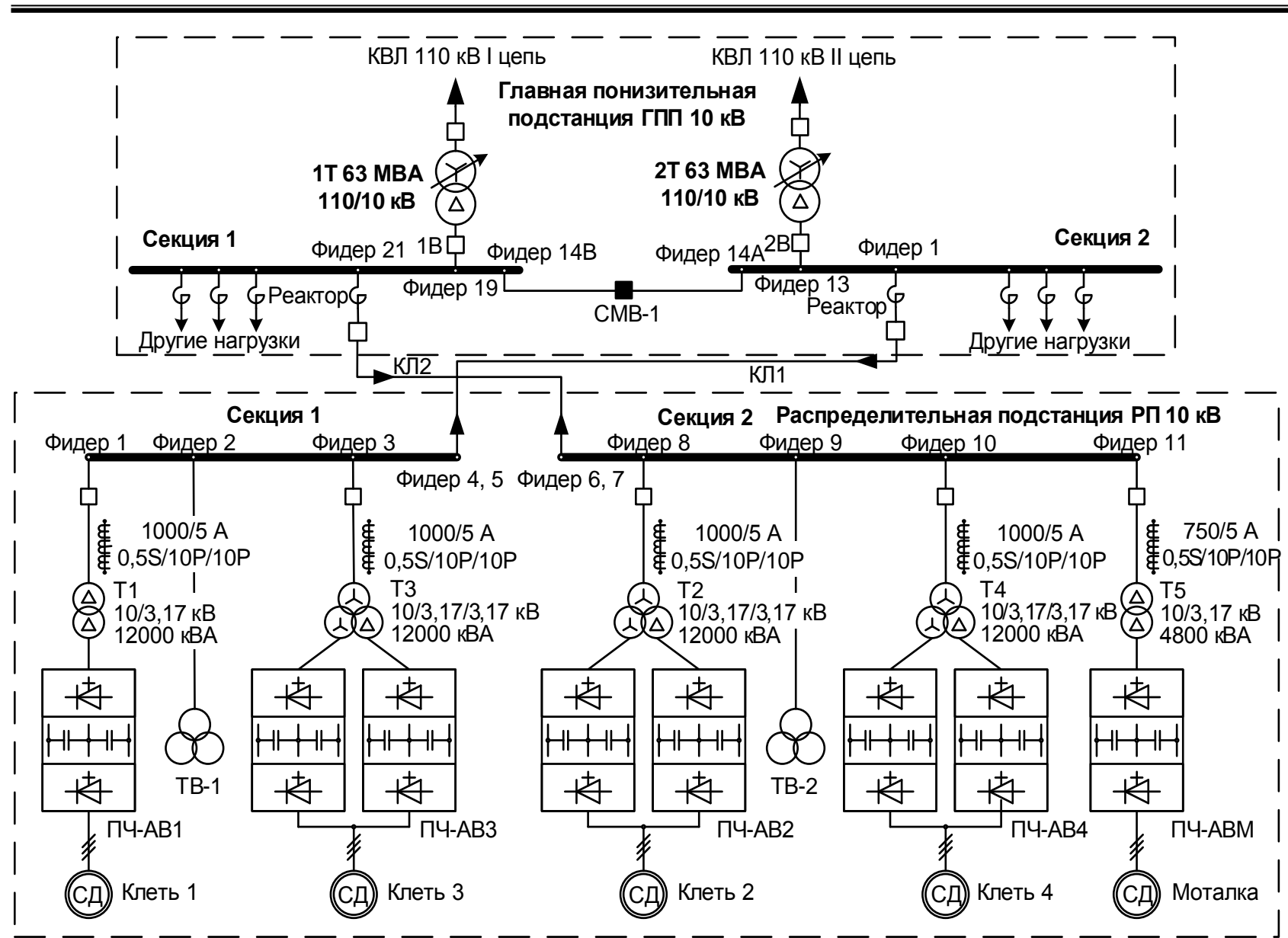

Рис. 1. Однолинейная схема распределительной подстанции, питающей электроприводы, на базе преобразователей частоты ABB серии ACS6000

Из-за использования токоограничивающих реакторов в ячейках РУ-10 кВ ГПП с индуктивными сопротивлениями $X_{\text {Р.ФАЗ }}=0,29-0,35$ Ом в частотных характеристиках относительно секций 1 и 2 ГПП, помимо основных резонансов в районе $f_{\text {РЕз }}=1450-2100$ Гц, присутствуют множественные резонансы. Эти резонансы вызваны взаимным влиянием токоограничивающих реакторов и суммарными емкостями кабелей отдельных фидеров ГПП. Так, при совместной работе секций ГПП с питанием от одного понизительного трансформатора 110/10 кВ 1Т или 2Т присутствуют дополнительные резонансы с частотами: 2650 Гц, 3150 Гц, 3650 Гц, 4050 Гц, 5150 Гц, 5750 Гц, 6350 Гц (рис. 2,б). Необходимо отметить, что амплитуды дополнительных резонансов в несколько раз ниже, чем у основных резонансных пиков в диапазоне $f_{\text {РЕ3 }}=1450-2100$ Гц. Это обусловливает незначительное усиление гармоник высокого порядка, генерируемых ПЧ с АВ в составе главных электроприводов четырехклетье- вого стана холодной прокатки. Иная ситуация на секциях РУ-10 кВ РП.

При работе электроприводов стана на секции 1 РУ-10 кВ РП наблюдается сильное искажение линейных напряжений (рис. 2,в,г). Суммарный коэфффициент гармонических составляющих напряжения $K_{U}$ превышает 17 \%. Доминирующими гармониками с амплитудой выше $4 \%$ относительно первой гармоники являются: 11,23 , 107, 109, (рис. 2,г). Гармонический состав напряжения на секции 2 РП отличается в худшую сторону по сравнению с секцией 1. Значение $K_{U}$ составляет 25,34 \% (рис. 2,д,е). Помимо значимой 23-й гармоники, гармонические составляющие напряжения высокого порядка с максимальными амплитудами смещены вправо по шкале частот: $n=121,131,133,135$. Данный фракт обусловлен смещением зоны резонанса в частотной характеристике в точке подключения электроприводов к секции 2 РУ-10 кВ РП с 4550-6550 Гц (91-я - 131-я гармоники) на 5450-7150 Гц (109-я - 143-я гармоники). 


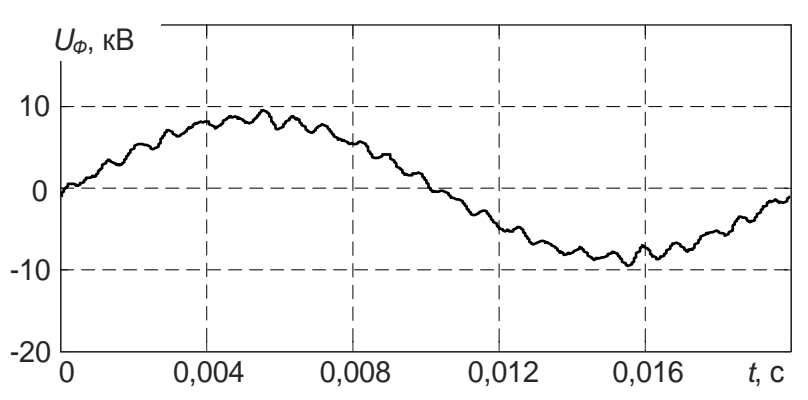

a)
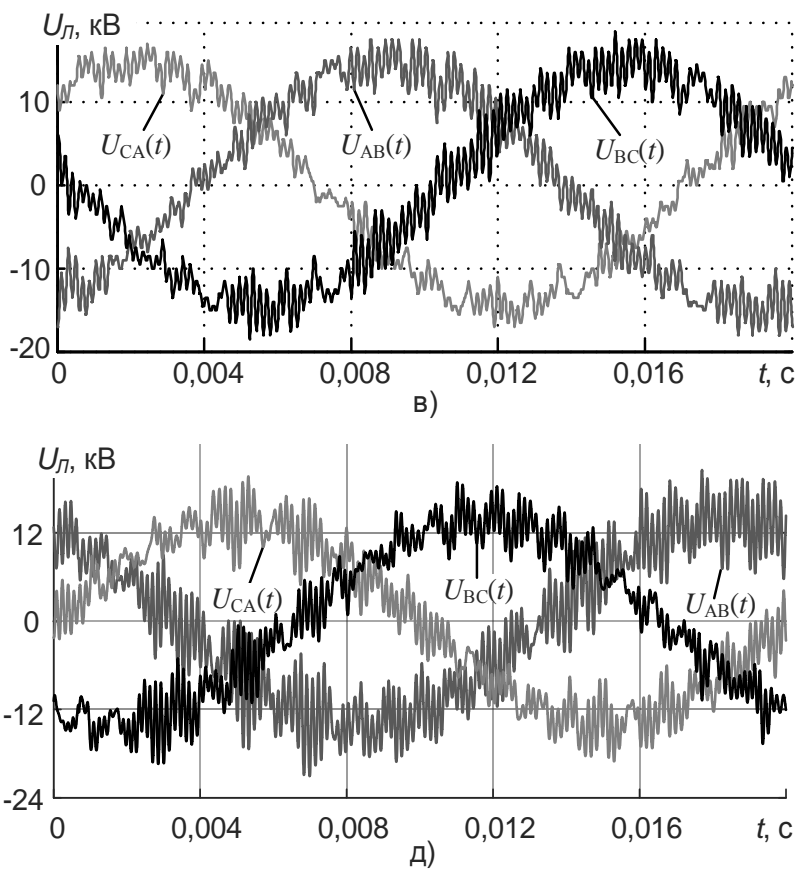

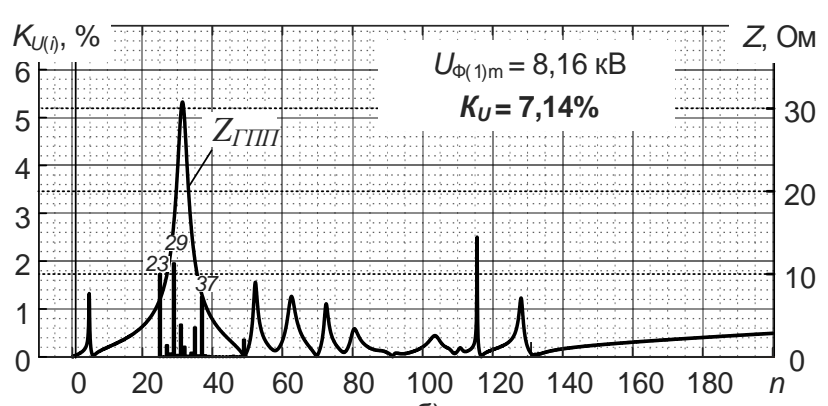

б)
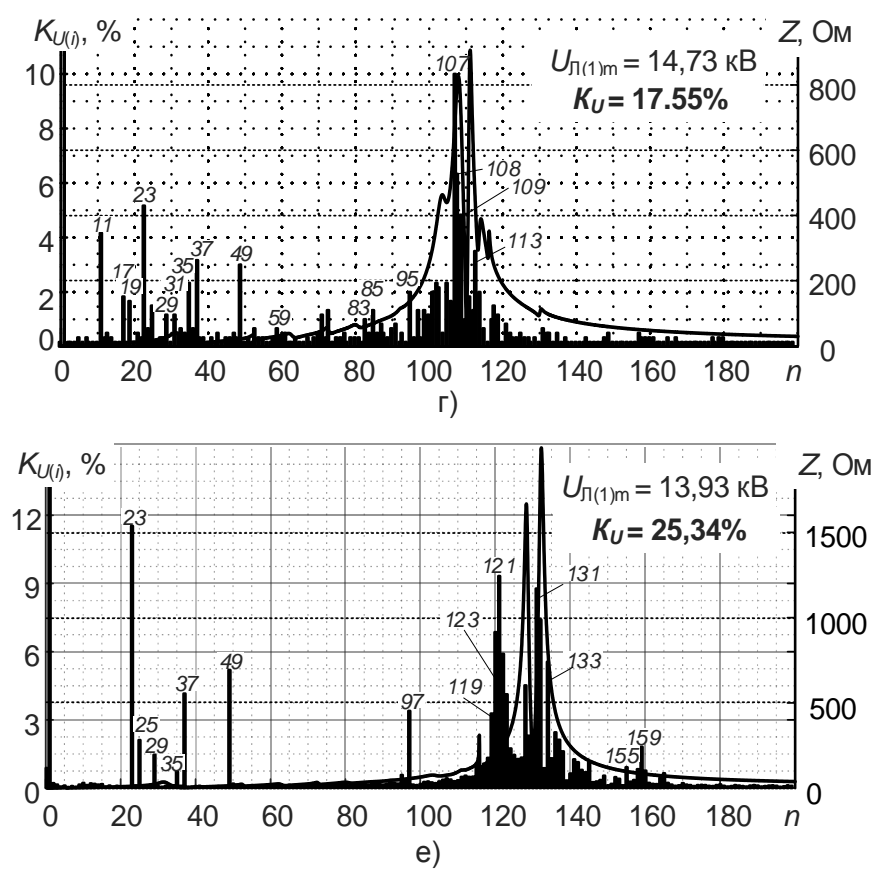

Рис. 2. Мгновенные значения напряжения, частотные характеристики и гармонический состав на шинах РУ-10 кВ: а, б - ГПП; в, г - секции 1 РП; д, е - секции 2 РП

Следует отметить, что характеристики $Z_{\mathrm{CETи}(f)}$ для секций 1 и 2 РУ-10 кВ РП значительно отличаются от частотной характеристики с множественными резонансами для секций РУ-10 кВ ГПП. Причиной этого является взаимное влияние емкостей кабельных линий, отходящих от фидеров 1 и 21 ГПП и индуктивностей токоограничивающих ректоров, расположенных в тех же ячейках. Характеристики $Z_{\text {СЕти(f) для секций }}$ 1 и 2 РП приведены на рис. 2,г,е. В харак-

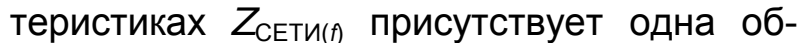
ласть подъема импеданса сети, расположенная в широком диапазоне частот: для секции 1 - от 4550 Гц $(n=91)$ до 6550 Гц ( $n=131)$ с максимумом около 5400 Гц ( $n=108)$; для секции 2 - от 5450 Гц $(n=109)$ до 7150 Гц $(n=143)$ с двумя максимумами 6100 Гц $(n=122)$ и 6600 Гц $(n=132)$. Амплитуды резонансных пиков составляют от 800 до 2000 Ом. Это является причиной усиления незначительных по амплитуде гармоник высокого порядка, генерируемых ПЧ с $\mathrm{AB}$, и сильного ухудшения качества напряжения на секциях РП.

С помощью программного обеспечения IBA PDA для проведения математического моделирования были записаны тренды основных параметров работы электроприводов стана (рис. 3), включающие величины угловых скоростей (рис. 3,a), моментов (рис. 3,б), выходных токов преобразователей (рис. 3,в), мощностей инвертеров (рис. 3,г) и токов активных выпрямителей (рис. 3,д). Полученные зависимости были аппроксимированы и использованы в комплексной имитационной модели системы электроснабжения района ГПП с учетом параметров питающей сети 110 кВ, сетевых трансформаторов 110/10 кВ, всех кабельных линий 10 кВ от ГПП, электроприводов стана, электроприемников других цеховых РП и подстанций. 


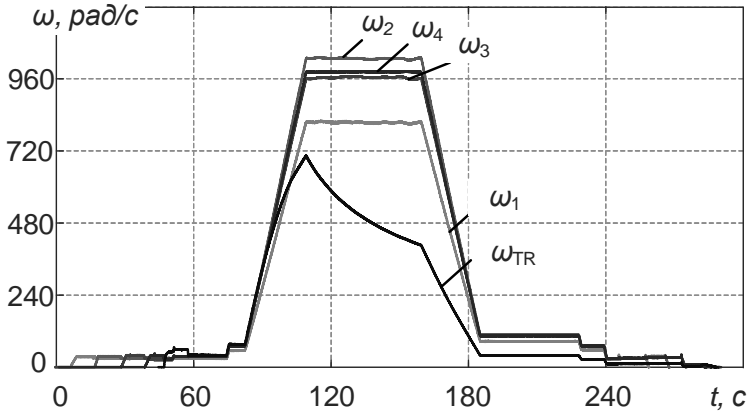

a)
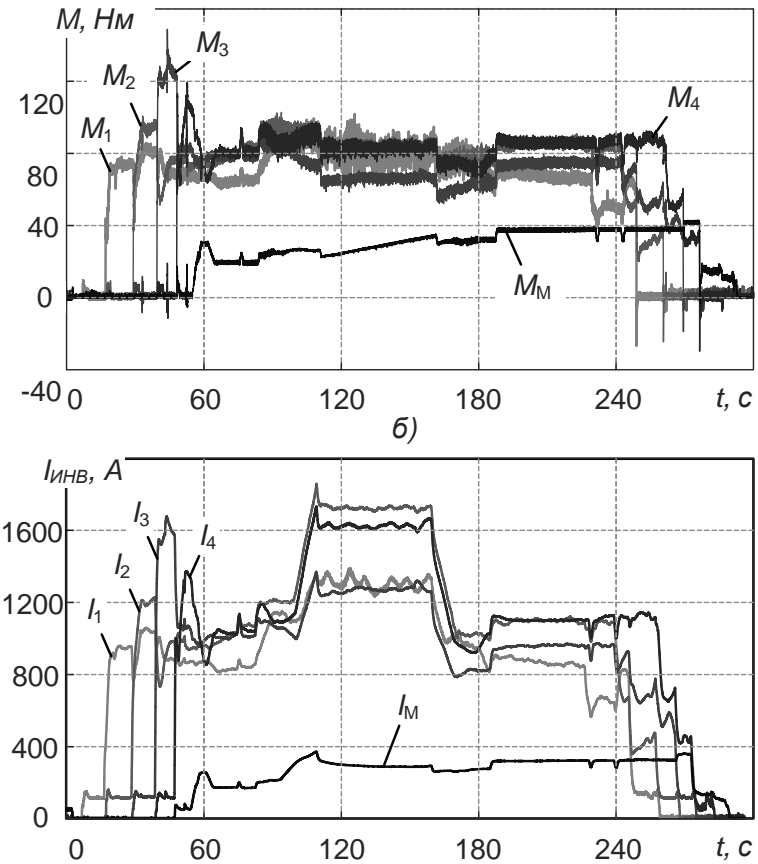

B)
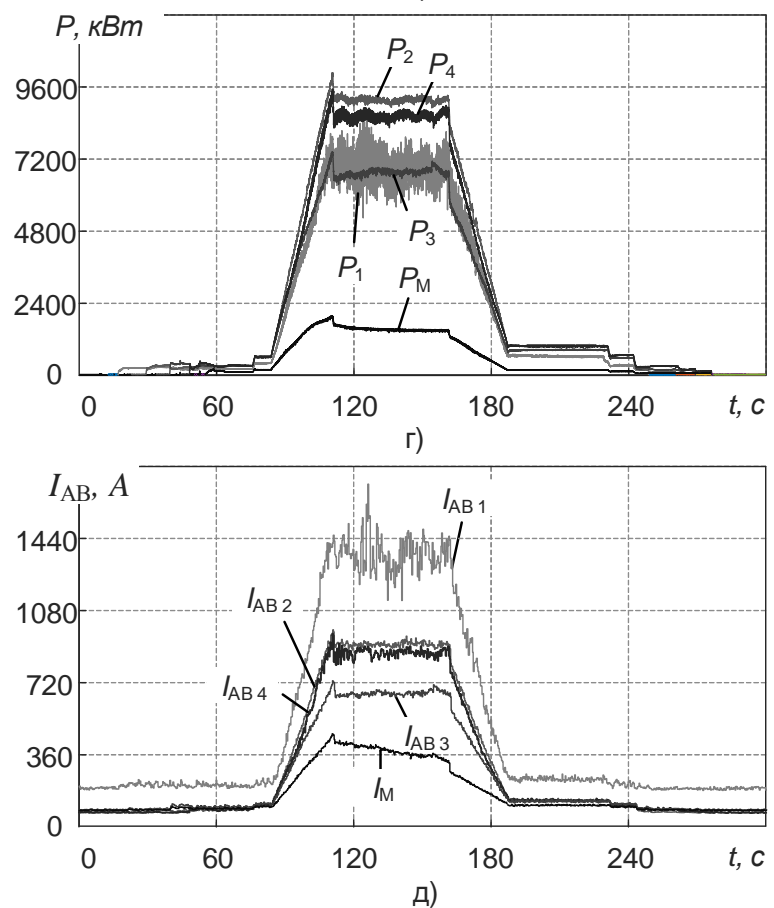

Рис. 3. Записанные мгновенные значения: а угловых скоростей; б - моментов; в - токов инверторов; г - мощностей; д - токов АВ электроприводов прокатного стана
На имитационной модели в программной среде Matlab Simulink были получены величины суммарного коэффицциента гармонических составляющих напряжения $K_{\cup}$ для секций 1 и 2 РУ-10 кВ главной понизительной подстанции ГПП при их совместной работе от 2 Т (рис. 4,a) и секций 1 и 2 РУ-10 кВ распределительной подстанции РП (рис. 4,б,в), питающей главные электроприводы стана. При имитационном моделировании использовались как исходные алгоритмы ШИМ АВ, так и усовершенствованные, предложенные в [5].

Результаты исследования. Анализ полученных результатов показал следующее. Применение усовершенствованного алгоритма ШИМ позволяет снизить уровень $K_{U}$ на шинах РУ-10 кВ секций 1 и 2 главной понизительной подстанции ГПП для всех режимов работы прокатного стана (рис. 4,a). В режиме заправки полосы в клети стана, а также выхода полосы с клетей на низкой скорости электроприводов значение $K_{U}$ составило в среднем 6,32 \% для исходного алгоритма и 2,82 \% для усовершенствованного. При прокатке значение $K_{U}$ составило 6,37 и 2,91\% соответственно.

Минимальные значения $K_{U}$ достигались во время разгона и торможения стана и составили 5,66 и 1,51\% соответственно. Максимальная величина $K_{U}$ для исходного алгоритма была достигнута в момент перед торможением стана и составила 6,44 \%, для усовершенствованного - сразу по завершению разгона и составила 3,00 \%.

Иной характер изменения величины $K_{U}$ наблюдается для напряжения на шинах секции 1 РУ-10 кВ РП (рис. 4,б), питающей электроприводы клети 1 и 3. В режиме заправки полосы в клети стана, а также выхода полосы с клетей на низкой скорости электроприводов значение $K_{U}$ составило в среднем $12,54 \%$ для исходного алгоритма и 11,63\% для усовершенствованного. После разгона электроприводов величина $K_{U}$ заметно снижается до 11,89 и 9,07 \% соответственно. При разгоне и торможении стана наблюдаются скачкообразные изменения величины $K_{U}$. Максимально значение $K_{U}$ было достигнуто при начале разгона стана и составило 13,61 и 12,21 \% соответственно. Минимум $K_{U}$ был достигнут в конце разгона и составил 11,57 и 8,54 \% соответственно. 


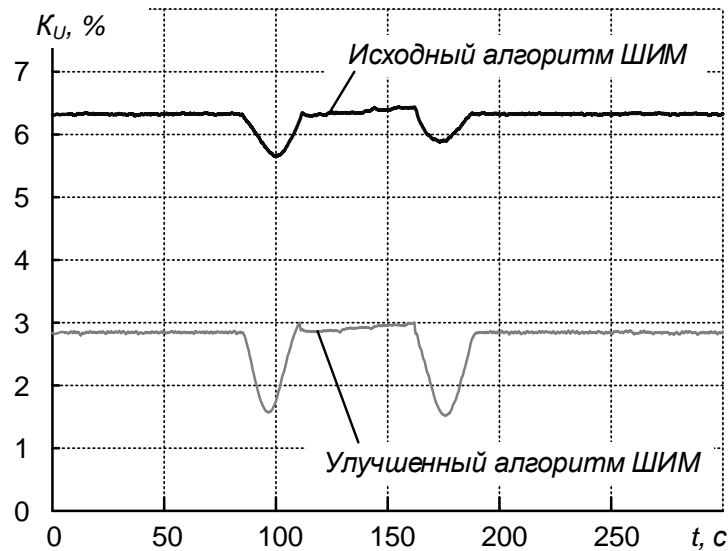

a)

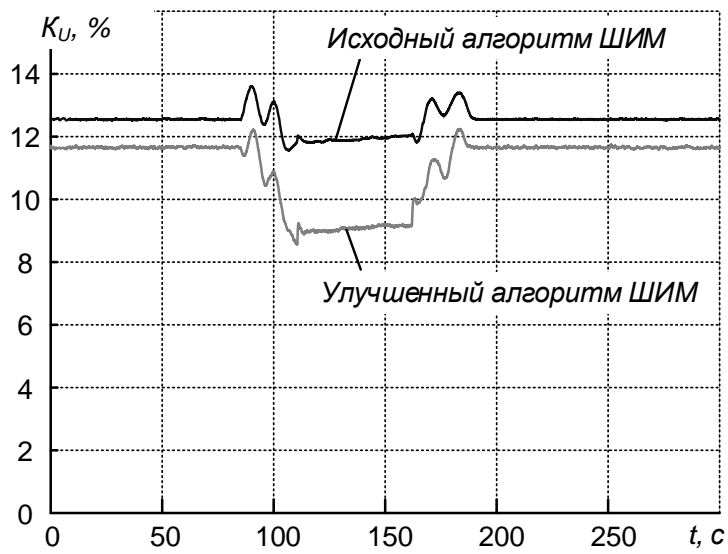

б)

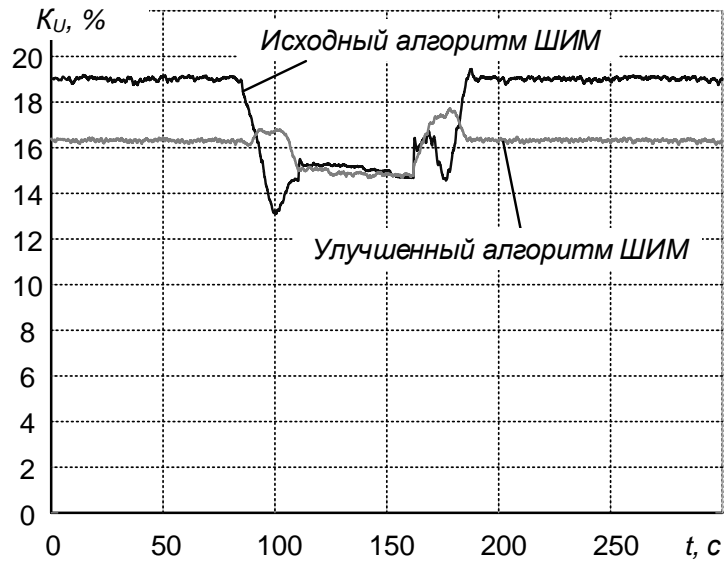

B)

Рис. 4. Полученные на имитационной модели величины $K_{U}$ для исходного и усовершенствованного алгоритмов ШИМ: а - для секций ГПП; б - для секции 1 РП; в - для секции 2 РП

Применение усовершенствованного алгоритма ШИМ АВ позволило снизить величину $K_{U}$ на шинах секции 2 РУ-10 кВ РП (рис. 4,в) при заправочной скорости клетей стана с 19,01 до 16,31\%. Однако при работе электроприводов стана на прокатной скорости эффрект от применения усовершенствованных алгоритмов в АВ ПЧ клетей 2, 4 и моталки практически незначителен: в среднем 15,19 и 14,89 \% соответственно. При использовании исходных ал- горитмов ШИМ при разгоне стана величина $K_{U}$ достигает минимума - в $13,12 \%$. При использовании усовершенствованных алгоритмов ШИМ при разгоне и торможении стана наблюдается рост величины $K_{U}$ для рассматриваемой секции. Максимальная величина составила 17,73 \%. Максимальная величина $K_{U}$ при использовании исходных алгоритмов ШИМ была получена сразу по завершении торможения стана после прокатки полосы и составила 19,44 \%. Минимальная величина $K_{U}$ при использовании усовершенствованных алгоритмов ШИМ АВ была получена во время прокатки полосы на установившейся скорости и составила 14,69 \%.

Для удобства анализа полученных результатов в таблицу сведены величины средних значений $K_{U}$ при работе стана на заправочной скорости и установившейся скорости при прокатке, а также минимальные и максимальные величины $K_{U}$ для секций РУ-10 кВ ГПП и секций 1 и 2 РУ-10 кВ РП.

\section{Результаты исследований}

\begin{tabular}{|c|c|c|c|}
\hline \multirow[b]{2}{*}{$\begin{array}{l}\text { Точка } \\
\text { измерения }\end{array}$} & \multirow[b]{2}{*}{ Описание } & \multicolumn{2}{|l|}{$K_{U}, \%$} \\
\hline & & $\begin{array}{l}\text { исход- } \\
\text { ный } \\
\text { алго- } \\
\text { ритм }\end{array}$ & $\begin{array}{l}\text { усовер- } \\
\text { вершен } \\
\text { шен- } \\
\text { ство- } \\
\text { ванный } \\
\text { алго- } \\
\text { ритм }\end{array}$ \\
\hline \multirow{4}{*}{$\begin{array}{l}\text { Секция } 1 \text { и } \\
2 \text { ГПП (па- } \\
\text { раллельная } \\
\text { работа от от } \\
2 \text { T) }\end{array}$} & $\begin{array}{l}\text { Заправочная } \\
\text { скорость }\end{array}$ & 6,32 & 2,82 \\
\hline & $\begin{array}{l}\text { Скорость } \\
\text { прокатки } \\
\end{array}$ & 6,37 & 2,91 \\
\hline & $\begin{array}{l}\text { Минимальная } \\
\text { величина }\end{array}$ & 5,66 & 1,51 \\
\hline & $\begin{array}{l}\text { Максимальная } \\
\text { величина }\end{array}$ & 6,44 & 3,00 \\
\hline \multirow{4}{*}{$\begin{array}{l}\text { Секция } 1 \\
\text { РП }\end{array}$} & $\begin{array}{l}\text { Заправочная } \\
\text { скорость }\end{array}$ & 12,54 & 11,63 \\
\hline & $\begin{array}{l}\text { Скорость } \\
\text { прокатки } \\
\end{array}$ & 11,89 & 9,07 \\
\hline & $\begin{array}{l}\text { Минимальная } \\
\text { величина }\end{array}$ & 11,57 & 8,54 \\
\hline & $\begin{array}{l}\text { Максимальная } \\
\text { величина }\end{array}$ & 13,61 & 12,21 \\
\hline \multirow{4}{*}{$\begin{array}{l}\text { Секция } 2 \\
\text { РП }\end{array}$} & $\begin{array}{l}\text { Заправочная } \\
\text { скорость }\end{array}$ & 19,01 & 16,31 \\
\hline & $\begin{array}{l}\text { Скорость } \\
\text { прокатки }\end{array}$ & 15,19 & 14,89 \\
\hline & $\begin{array}{l}\text { Минимальная } \\
\text { величина }\end{array}$ & 13,12 & 14,69 \\
\hline & $\begin{array}{l}\text { Максимальная } \\
\text { величина }\end{array}$ & 19,44 & 17,73 \\
\hline
\end{tabular}


Анализ полученных результатов показывает, что применение усовершенствованных алгоритмов ШИМ АВ позволяет добиться улучшения качества напряжения как на секциях распределительной подстанции РП, питающей электроприводы прокатного стана, так и на секциях главной понизительной подстанции ГПП системы внутризаводского электроснабжения, являющихся общей точкой для всех потребителей, что наиболее важно. Однако стоит отметить, что в единственном случае - для секции 2 РП - при применении исходных алгоритмов ШИМ в режиме разгона стана достигается минимум величины $K_{U}$, который составляет $13,12 \%$, что превосходит показатели усовершенствованного алгоритма (14,69 \%). Но это единственный кратковременный момент (в сумме $\sim 30$ с) в цикле работы прокатного стана, когда исходные алгоритмы ШИМ AB превосходят представленные усовершенствованные.

Выводы. По результатам проведенных исследований было показано значимое влияние режимов работы электропривода прокатного стана на качество электроэнергии распределительной сети 10 кВ системы внутризаводского электроснабжения металлургического предприятия. Исследования показали, что коэффрициент $K_{U}$ на секциях 10 кВ главной понизительной подстанции изменяется нелинейным образом в динамических режимах разгона и торможения электроприводов. Характер изменения коэффрициента $K_{U}$ также зависит от наличия резонансных явлений в частотной характеристике питающей сети среднего напряжения. Данное обстоятельство доказывает необходимость учета режимов работы электроприводов при исследовании влияния электроприводов с ПЧ-АВ на качество напряжения питающей сети при проведении экспериментальных исследований на действующем оборудовании предприятий, а также при выполнении теоретических исследований на математических моделях.

При разработке усовершенствованных алгоритмов ШИМ активных выпрямителей с функцией адаптации к резонансным явлениям в частотной характеристике питающей сети должна проводиться дополнительная проверка эфффективности алгоритмов для всего цикла работы электроприводов.

Для рассматриваемой системы электроснабжения прокатного стана разрабо- танные усовершенствованные алгоритмы ШИМ обеспечивают достижение значимого технического эффректа по уменьшению значения $K_{U}$ как на секциях 10 кВ главной понизительной подстанции предприятия, так и на секциях цехового распределительного устройства, от которого получают питание мощные электроприводы прокатных клетей и моталки. Максимальная величина относительного изменения $\delta K_{U}$ на секциях ГПП составила $-55,4$ \%, при этом на секциях цехового РУ $-10 \kappa \mathrm{B} \delta K_{U}=-23,7 \%$.

\section{Список литературы}

1. Retrofit of a Hot Rolling Mill Plant With Three-Level Active Front End Drives / G.A. Orcajo, J.R. Diez, J.M.C. Rodriguez, et al. // IEEE Transactions on Industry Applications. - May-June 2018. - Vol. 54, no. 3. - P. 2964-2974. DOI: 10.1109/TIA.2018.2808159.

2. Храмшин Т.Р., Корнилов Г.П., Крубцов Д.С. Оценка методов широтно-импульсной модуляции напряжения активных выпрямителей прокатных станов // Машиностроение: сетевой электронный научный журнал. - 2013. - № 2. C. 48-53.

3. Reactive power compensation in industrial grid via high-power adjustable speed drives with medium voltage 3L-NPC BTB converters / A.A. Radionov, V.R. Gasiyarov, A.S. Maklakov, E.A. Maklakova // International Journal of Power Electronics and Drive Systems. - 2017. - Vol. 8(4). P. 1455-1466.

4. Маклаков А.С. Имитационное моделирование главного электропривода прокатной клети толстолистового стана 5000 // Машиностроение: сетевой электронный научный журнал. - 2014. № 3. - C. $16-25$.

5. Providing Electromagnetic Compatibility of High-Power Frequency Converters with Active Rectifiers at Internal Power Supply System of Cherepovets Steel Mill / A.A. Nikolaev, I.G. Gilemov, M.V. Bulanov, V.I. Kosmatov // 2021 XVIII International Scientific Technical Conference Alternating Current Electric Drives (ACED). - 2021. P. 1-8. DOI: 10.1109/ACED50605.2021.9462264.

6. Маклаков А.С., Гасияров В.Р., Белый А.В. Энергосберегающий электропривод на базе двухзвенного преобразователя частоты с активным выпрямителем и автономным инвертором напряжения // Электротехника: сетевой электрон. науч. журнал. - 2014. - Т. 1 , № 1. - C. 23-30.

7. O'Brien K., Teichmann R., Bernet S. Active rectifier for medium voltage drive systems // Applied Power Electronics Conference and Exposition, 2001. APEC 2001. Sixteenth Annual IEEE. - 2001. P. 557-562. 
8. Research on hybrid SHEPWM based on different switching patterns / T. Jing, A. Maklakov, A. Radionov, et al. // International Journal of Power Electronics and Drive Systems. - 2019. - Vol. 10(4). P. 1875-1884.

9. Franquelo L.G., Nápoles J. A Flexible Selective Harmonic Mitigation Technique to Meet Grid Codes in Three-Level PWM Converters // IEEE Transactions on Industrial Electronics. - December 2007. - Vol. 54, no. 6.

10. Moeini A., Zhao H., Wang S. A current reference based selective harmonic current mitigation pwm technique to improve the performance of cascaded $\mathrm{H}$-bridge multilevel active rectifiers // IEEE Trans. Ind. Electronics. - 2018. Vol. 65. - P. 727-737.

11. Jing T., Maklakov A., Radionov A. Two Selective Harmonic Control Techniques Applied in 10kV Grid with Three-Level NPC Inverter // 2019 IEEE Russian Workshop on Power Engineering and Automation of Metallurgy Industry: Research \& Practice (PEAMI), Magnitogorsk, Russia. - 2019. P. 75-79. DOI: 10.1109/PEAMI.2019.8915413.

12. Nikolaev A.A., Bulanov M.V., Antropova L.I. Ways to ensure electromagnetic compatibility of powerful frequency converters in internal power supply systems of industrial enterprises in the presence of resonance phenomena // International Conference on Industrial Engineering, Applications and Manufacturing. -2019.

13. Экспериментальные исследования электромагнитной совместимости современных электроприводов в системе электроснабжения металлургического предприятия / А.А. Николаев, Г.П. Корнилов, Т.Р. Храмшин и др. // Вестник Магнитогорского государственного технического университета им. Г.И. Носова. - 2016. - Т. 14, № 4. - C. 96-105. DOI:10.18503/1995-2732-201614-4-96-105.

14. Nikolaev A.A., Gilemov I.G. Improvement of Power Quality in Supply Network with Active Rectifiers by Selecting Optimal Switching Angle Tables of PWM // Elektrotekhnicheskie sistemy i kompleksy [Electrotechnical Systems and Complexes]. - 2019. - No. 4(45). - P. 35-42. (In Russian). DOI: 10.18503/2311-8318-2019-4(45)35-42.

15. Endrejat F., Pillay P. Resonance Overvoltages in Medium Voltage Multilevel Drive System // 2007 IEEE International Electric Machines \& Drives Conference - Antalya. - 2007. P. 736-741.

16. Harmonic Analysis of Grid-tied Active Front End Inverters for the Frequency Range of 0-9 kHz in Distribution Networks:Addressing Future Regulations / H. Rathnayake, K.G. Khajeh, F. Zare, R. Sharma // 2019 IEEE International Conference on Industrial Technology (ICIT). - 2019. P. 446-451. DOI: 10.1109/ICIT.2019.8755015.

17. Маклаков А.C., Радионов А.A. Исследование векторной ШИМ с различными таблицами переключения силовых ключей трехуровневого преобразователя // Электротехника: сетевой электронный научный журнал. 2015. - T. 2, № 1. - С. 30-37.

18. loffe I., lunusov R., Kostylev A. The Comparative Analysis of Processes in Active Front End (AFE) for Cases of Different Power Sources // 2019 IEEE Russian Workshop on Power Engineering and Automation of Metallurgy Industry: Research \& Practice (PEAMI). - 2019. - P. 99-104. DOI: 10.1109/PEAMI.2019.8915097.

\section{References}

1. Orcajo, G.A., Diez, J.R., Rodriguez, J.M.C., Norniella, J.G. Retrofit of a Hot Rolling Mill Plant With Three-Level Active Front End Drives. IEEE Transactions on Industry Applications, 2018 MayJune, vol. 54, no. 3, pp. 2964-2974. DOI: 10.1109/TIA.2018.2808159.

2. Khramshin, T.P., Kornilov, G.P., Krubtsov, D.S. Otsenka metodov shirotnoimpul'snoy modulyatsii napryazheniya aktivnykh vypryamiteley prokatnykh stanov [Evaluation of methods of PWM voltage active rectifiers rolling mills]. Mashinostroenie: setevoy elektronnyy nauchnyy zhurnal, 2013, no. 2, pp. 48-52.

3. Radionov, A.A., Gasiyarov, V.R., Maklakov, A.S., Maklakova, E.A. Reactive power compensation in industrial grid via high-power adjustable speed drives with medium voltage 3L-NPC BTB converters. International Journal of Power Electronics and Drive Systems, 2017, vol. 8(4), pp. 1455-1466.

4. Maklakov, A.S. Imitatsionnoe modelirovanie glavnogo elektroprivoda prokatnoy kleti tolstolistovogo stana 5000 [Simulation of the main electric drive of the plate mill rolling stand]. Mashinostroenie: setevoy elektronnyy nauchnyy zhurnal, 2014, no. 3, pp. 16-25.

5. Nikolaev, A.A., Gilemov, I.G., Bulanov, M.V., Kosmatov, V.I. Providing Electromagnetic Compatibility of High-Power Frequency Converters with Active Rectifiers at Internal Power Supply System of Cherepovets Steel Mill. 2021 XVIII International Scientific Technical Conference Alternating Current Electric Drives (ACED), 2021, pp. 1-8. DOI: 10.1109/ACED50605.2021.9462264.

6. Maklakov, A.S., Gasiyarov, V.R., Belyy, A.V. Energosberegayushchiy elektroprivod na baze dvukhzvennogo preobrazovatelya chastoty $s$ aktivnym vypryamitelem i avtonomnym invertorom napryazheniya [Energy-saving electric drive based on a two-link frequency converter with an active rectifier and an autonomous voltage inverter]. Elektrotekhnika: setevoy elektronnyy nauchnyy zhurnal, 2014, vol. 1, no. 1, pp. 23-30.

7. O'Brien, K., Teichmann, R., Bernet, S. Active rectifier for medium voltage drive systems. Applied Power Electronics Conference and Exposition, 2001. APEC 2001. Sixteenth Annual IEEE, 2001, pp. 557-562. 
8. Jing, T., Maklakov, A., Radionov, A., Baskov, S., Kulmukhametova, A. Research on hybrid SHEPWM based on different switching patterns. International Journal of Power Electronics and Drive Systems, 2019, vol. 10(4), pp. 1875-1884.

9. Franquelo, L.G., Nápoles, J. A Flexible Selective Harmonic Mitigation Technique to Meet Grid Codes in Three-Level PWM Converters. IEEE Transactions on Industrial Electronics, 2007 December, vol. 54, no. 6 .

10. Moeini, A., Zhao, H., Wang, S. A current reference based selective harmonic current mitigation pwm technique to improve the performance of cascaded $\mathrm{H}$-bridge multilevel active rectifiers. IEEE Trans. Ind. Electronics, 2018, vol. 65, pp. 727-737.

11. Jing, T., Maklakov, A., Radionov, A. Two Selective Harmonic Control Techniques Applied in 10kV Grid with Three-Level NPC Inverter. 2019 IEEE Russian Workshop on Power Engineering and Automation of Metallurgy Industry: Research \& Practice (PEAMI), Magnitogorsk, Russia, 2019, pp. 75-79. DOI: 10.1109/PEAMI.2019.8915413.

12. Nikolaev, A.A., Bulanov, M.V., Antropova, L.I. Ways to ensure electromagnetic compatibility of powerful frequency converters in internal power supply systems of industrial enterprises in the presence of resonance phenomena. International Conference on Industrial Engineering, Applications and Manufacturing, 2019.

13. Nikolaev, A.A., Kornilov, G.P., Khramshin, T.R., Nikiforov, G., Mutallapova, F.F. Eksperimental'nye issledovaniya elektromagnitnoy sovmestimosti sovremennykh elektroprivodov $\mathrm{v}$ sisteme elektrosnabzheniya metallurgicheskogo predpriyatiya [Experimental studies of electromagnetic compatibility of modern electric drives in the power supply system of industrial enterprises]. Vestnik Magnitogorskogo gosudarstvennogo tekhnicheskogo universiteta im. G.I. Nosova, 2016, vol. 14, no. 4, pp. 96-105.

14. Nikolaev, A.A., Gilemov, I.G. Improvement of Power Quality in Supply Network with Active Rectifiers by Selecting Optimal Switching Angle Tables of PWM. Elektrotekhnicheskie sistemy i kompleksy, 2019, no. 4(45), pp. 35-42. DOI: 10.18503/2311-8318-2019-4(45)-35-42.

15. Endrejat, F., Pillay, P. Resonance Overvoltages in Medium Voltage Multilevel Drive System. 2007 IEEE International Electric Machines \& Drives Conference. Antalya, 2007, pp. 736-741.

16. Rathnayake, H., Khajeh, K.G., Zare, F., Sharma, R. Harmonic Analysis of Grid-tied Active Front End Inverters for the Frequency Range of 0-9 kHz in Distribution Networks: Addressing Future Regulations. 2019 IEEE International Conference on Industrial Technology (ICIT), 2019, pp. 446-451. DOI: 10.1109/ICIT.2019.8755015.

17. Maklakov, A.S., Radionov, A.A. Issledovanie vektornoy ShIM s razlichnymi tablitsami pereklyucheniya silovykh klyuchey trekhurovnevogo preobrazovatelya [Study of SVPWM with Various Vector Selection Tables of Three Level Converter]. Elektrotekhnika: setevoy elektronnyy nauchnyy zhurnal, 2015, vol. 2, no. 1, pp. 30-37.

18. loffe, I., lunusov, R., Kostylev, A. The Comparative Analysis of Processes in Active Front End (AFE) for Cases of Different Power Sources. 2019 IEEE Russian Workshop on Power Engineering and Automation of Metallurgy Industry: Research \& Practice (PEAMI), 2019, pp. 99-104. DOI: 10.1109/PEAMI.2019.8915097. 